\title{
Inflammatory response is modulated by lincRNACox2 via the NF-кB pathway in macrophages infected by Mycobacterium tuberculosis
}

\author{
DANYE LI ${ }^{1 *}$, CAIYAN GAO $^{2 *}$, LING ZHAO $^{3}$ and YONGMING ZHANG ${ }^{1}$ \\ ${ }^{1}$ Department of Pulmonary and Critical Care Medicine, China-Japan Friendship Hospital, Beijing 100029; \\ ${ }^{2}$ Department of Respiration, Hongda Hospital of Jiamusi University, Jiamusi, Heilongjiang 154004; \\ ${ }^{3}$ Department of Pathology, China-Japan Friendship Hospital, Beijing 100029, P.R. China
}

Received November 19, 2019; Accepted February 26, 2020

DOI: $10.3892 / \mathrm{mmr} .2020 .11053$

\begin{abstract}
Long intergenic non-coding RNAs (lincRNAs) are long non-coding transcripts from the intergenic regions of annotated protein-coding genes. lincRNA cyclooxygenase 2 (Cox2) is an early-primary response gene regulated by the $\mathrm{NF}-\kappa \mathrm{B}$ signaling pathway in macrophages. It was found that lincRNACox 2 was significantly increased in patients with the Mycobacterium tuberculosis (M. tuberculosis) H37Ra strain infection and macrophages, using reverse transcription-quantitative PCR (RT-qPCR). ELISA, western blotting and RT-qPCR results indicated that the inflammatory response factors tumor necrosis factor- $\alpha$, interferon- $\gamma$, interleukin-6, Cox 2 and inducible nitric oxide synthase were significantly increased in H37Ra infected macrophages. In addition, the inflammatory regulating proteins $\mathrm{NF}-\kappa \mathrm{B}$ and Stat 3 were significantly increased in $\mathrm{H} 37 \mathrm{Ra}$ infected macrophages but decreased in lincRNACox 2 knockdown macrophages infected with H37Ra. Moreover, the knockdown of lincRNACox 2 increased the apoptotic rate of H37Ra infected macrophages and facilitated the proliferation of H37Ra. Collectively, the present results suggested that lincRNACox 2 may be required for the activation of $\mathrm{NF}-\kappa \mathrm{B}$ and Stat3, in order to regulate inflammatory responses involved in resistance to $M$. tuberculosis infection.
\end{abstract}

\section{Introduction}

Mycobacterium tuberculosis (M. tuberculosis) is the causative agent of tuberculosis (TB) and is a major public health threat

Correspondence to: Dr Yongming Zhang, Department of Pulmonary and Critical Care Medicine, China-Japan Friendship Hospital, 2 East Yinghua Road, Chaoyang, Beijing 100029, P.R. China E-mail: zhangyongmingz19@163.com

${ }^{*}$ Contributed equally

Key words: lincRNACox2, Mycobacterium tuberculosis, macrophages, $\mathrm{NF}-\kappa \mathrm{B}$ associated with high morbidity and mortality rates worldwide (1). Currently, $>30 \%$ of the population worldwide is infected with M. tuberculosis according to data from the World Health Organization (2). The host immune response against M.tuberculosis is complex and multifaceted, thus understanding the pathogenesis and host immune mechanisms against TB will facilitate the development of novel diagnostic and therapeutic strategies for TB (3). Macrophages are critical immune cells that play important roles in the host immune system by phagocytizing M. tuberculosis to help eliminate infection, as well as initiating the protective immune responses by presenting antigens to $\mathrm{T}$ lymphocytes $(4,5)$. Macrophages can prevent the intracellular growth and persistence of M.tuberculosis during various phases of $\mathrm{TB}$, from primary infection with bacillary dissemination (6). These previous studies have revealed the functions of macrophages in M. tuberculosis infection, but the underlying regulatory mechanisms are still not fully understood.

Long non-coding RNAs (lncRNAs) are transcripts $\sim 200$ nucleotides in length that regulate the expression of protein encoding genes at the transcriptional and post-transcriptional levels (7). By dysregulating target genes, IncRNAs can participate in the development and progression of various human diseases, including cancer, inflammation and autoimmune diseases (8-10). The regulatory potential of lncRNAs-in epigenetic reprograming is emerging as a novel mechanism to explain functional plasticity and the diversity of immune cells including $\mathrm{T}$ cells, B cells, dendritic cells and macrophages. Previous studies have shown that lncRNAs also play an essential role in human infectious diseases such as TB $(11,12)$. Pawar et al $(13)$ showed that downregulation of the IncRNA maternally expressed 3 promotes the eradication of intracellular mycobacterium in $M$. bovis Bacille Calmette Guerin-infected macrophages.

The aim of the present study was to investigate the regulatory mechanisms of the inflammatory response modulated by long intergenic non-coding RNA cyclooxygenase 2 (lincRNACox2) in M. tuberculosis H37Ra infected macrophages.

\section{Materials and methods}

Samples collection and ethical statement. The peripheral blood samples $(3 \mathrm{ml})$ were obtained from 56 patients with 
active pulmonary TB (age range, 22-62 years; median age, 36 years; sex, male: female $=3: 5$ ) and 60 healthy individuals (age range, 20-60 years; median age, 32 years; male:female $=4: 6$ ) in the China-Japan Friendship Hospital from June 2015 to June 2017. Written informed consent was obtained from all donors prior to the study. This study was approved by the Ethics Committee of the China-Japan Friendship Hospital (grant no. ZRYYEC/2015/27-2).

Cell culture. THP-1 cells were purchased from the American Type Culture Collection and cultured in RPMI-1640 (Gibco; Thermo Fisher Scientific, Inc.) with 10\% FBS (Gibco; Thermo Fisher Scientific, Inc) and antibiotics $(100 \mathrm{U} / \mathrm{ml}$ penicillin and $100 \mathrm{mg} / \mathrm{ml}$ streptomycin) at $37^{\circ} \mathrm{C}$ under the humidified atmosphere of $5 \% \mathrm{CO}_{2}$. The attenuated M. tuberculosis strain H37Ra was provided by the China Center for Disease Control. M. tuberculosis H37Ra was cultured on Middlebrooks 7H9 plates. M. tuberculosis H37Ra culture and related experiments were performed in the BSL-3 (P3) Laboratory of China Center for Disease Control.

THP-1 cells were differentiated into macrophages using Phorbol ester $(0.01 \mathrm{mM}$; Merck KGaA) for $24 \mathrm{~h}$ and were then incubated with H37Ra at a multiplicity of infection of 10 (10 bacteria: 1 cell) for $24 \mathrm{~h}$ at $37^{\circ} \mathrm{C}$.

Small interfering RNA (siRNA) transfection and luciferase reporter assay. For gene silencing, siRNAs for lincRNACox 2 were synthesized and purchased from Invitrogen (Thermo Fisher Scientific, Inc.). The following siRNAs were used: Mock siRNA sense, 5'-UAAGGCUAU GAAGAGAUACUU-3' and antisense 5'-GUAUCUCUUCAU AGCCUUAUU-3'; si-lincRNACox 2 siRNA sense, 5'-GCC CUAAUA AGUGGGUUGUUU-3' and antisense, 5'-ACA ACCCACUUAUUAGGGCUU-3'; and lincRNACox2 sense, 5'-AGTATGGGATAACCAGCTGAGGT-3' and antisense, 5'-GAATGCTGAGAGTGGGAGAAATAG-3'. Macrophages were transfected with siRNAs (final concentration, $20 \mathrm{nM}$ ) using Lipofectamine RNAiMax reagent (cat. no. 13778030; Thermo Fisher Scientific, Inc.) according to the manufacturer's protocol for $24 \mathrm{~h}$. The lincRNACox 2 expression vector was generated by reverse transcription-quantitative PCR (RT-qPCR) amplification of lincRNACox 2 cDNA using RNA from macrophages and cloned into a pcDNA3.3 vector (Thermo Fisher Scientific, Inc.). The promoter of lincRNACox 2 was amplified by PCR from human macrophage genomic DNA. Macrophages were transfected with each reporter construct for $24 \mathrm{~h}$, followed by assessment of luciferase activity.

$R T-q P C R$ and rapid amplification of cDNA ends (RACE) $P C R$. Total RNA was extracted with TRIzol solution (cat.no. R0016; Beyotime Institute of Biotechnology) according to the manufacturer's protocol. All primers for PCR analysis were designed and synthesized by Invitrogen (Thermo Fisher Scientific, Inc.). RT-qPCR was performed on a Master-cycler (Eppendorf Corp.) using the SYBR Green PCR Master Mix [cat. no. R10T1; Takara Biotechnology (Dalian) Co., Ltd.]. The PCR conditions were as follows: Initial denaturation at $95^{\circ} \mathrm{C}$ for $25 \mathrm{sec}$, followed by 36 cycles at $95^{\circ} \mathrm{C}$ for $20 \mathrm{sec}, 58^{\circ} \mathrm{C}$ for $20 \mathrm{sec}$ and $72^{\circ} \mathrm{C}$ for $15 \mathrm{sec}$. U6 was used as an internal control and the relative expression of genes was calculated with the $2^{-\Delta \Delta \mathrm{Cq}}$ method (14). Primers used were as follows: lincRNACox2 forward, 5'-AGTATGGGATAACCAGCTGAG GT-3' and reverse, 5'-GAATGCTGAGAGTGGGAGAAA TAG-3'; and U6 forward, 5'-CGCTTCGGCACATATACTA-3' and reverse, 5'-CGCTTCACGAATTTGCGTGTCA-3'.

RACE PCR was used to identify the $5^{\prime}$ and $3^{\prime}$ ends of lincRNACox 2 to localize the transcriptional start site. The SMART RACE cDNA Amplification kit (cat. no. 634923; Clontech Laboratories, Inc.) was used and the primers for $5^{\prime}$ and $3^{\prime}$ ends of lincRNACox 2 RACE PCR analysis were designed and synthesized by Invitrogen (Thermo Fisher Scientific, Inc.).

ELISA assay. ELISA kits were used to detect the levels of the cytokines tumor necrosis factor (TNF)- $\alpha$ (cat. no. EK0526; Wuhan Boster Biological Technology, Ltd.), interferon (IFN)- $\gamma$ (cat. no. EK0373; Wuhan Boster Biological Technology, Ltd.) and interleukin (IL)-6 (cat. no. EK0411; Wuhan Boster Biological Technology, Ltd.) in the plasma of patients with TB infection and in cell supernatant of macrophages infected with H37Ra, according to the manufacturer's protocol. Cytokine concentrations were calculated using a standard curve.

Western blotting. Cell lysates were obtained with RIPA lysis buffer (cat. no. P0013B; Beyotime Institute of Biotechnology) and were quantified using a bicinchoninic acid kit (cat. no. P0012S; Beyotime Institute of Biotechnology). Proteins $(30 \mu \mathrm{g})$ were run on $10 \%$ SDS-PAGE and transferred to PVDF membranes (EMD Millipore). After blocking with $5 \%$ non-fat milk for $1 \mathrm{~h}$ at room temperature, the membranes were incubated overnight at $4^{\circ} \mathrm{C}$ with the following antibodies according to the manufacturer's protocol: Cox 2 (cat. no. sc1745; Santa Cruz Biotechnology, Inc.; 1:800), inducible nitric oxide synthase (iNOS; cat. no. sc-7271; Santa Cruz Biotechnology, Inc.; 1:1,200), Stat3 (cat. no. sc-8019; Santa Cruz Biotechnology, Inc.; 1:600), NF-kB (cat. no. sc-8414; Santa Cruz Biotechnology, Inc.; 1:1,000) and GAPDH (cat. no. sc-47724; Santa Cruz Biotechnology, Inc.; 1:2,000). Then, membranes were incubated with the goat anti-rabbit immunoglobin G-horseradish peroxidase (cat. no. sc-2004; 1:4,000; Santa Cruz Biotechnology, Inc.). Specific protein bands were detected using the Chemiluminescent Substrate system (Thermo Fisher Scientific, Inc.). The band intensity was measured using ImageJ 1.47 (National Institutes of Health).

Analysis of the apoptotic rate of H37Ra infected macrophages. Macrophages transfected with si-lincRNACox 2 or lincRNACox 2 were infected with $1 \times 10^{5}$ colony forming units (CFU) of $\mathrm{H} 37 \mathrm{Ra}$ for $24 \mathrm{~h}$ at $37^{\circ} \mathrm{C}$ and then cultured for $24 \mathrm{~h}$ in medium at $37^{\circ} \mathrm{C}$. Then, cells were harvested and incubated with Annexin-V/PI (cat. no. C1062L; Beyotime Institute of Biotechnology) for $30 \mathrm{~min}$ in the dark on ice at $4^{\circ} \mathrm{C}$. Apoptotic cells were detected on a DxFLEX flow cytometer (Beckman Coulter, Inc.) and analyzed using CytExpert version 2 software (Beckman Coulter, Inc.), according to the manufacturer's protocol.

Proliferation analysis of H37Ra. Macrophages were transfected with si-lincRNACox 2 or lincRNACox 2 for $24 \mathrm{~h}$, then 

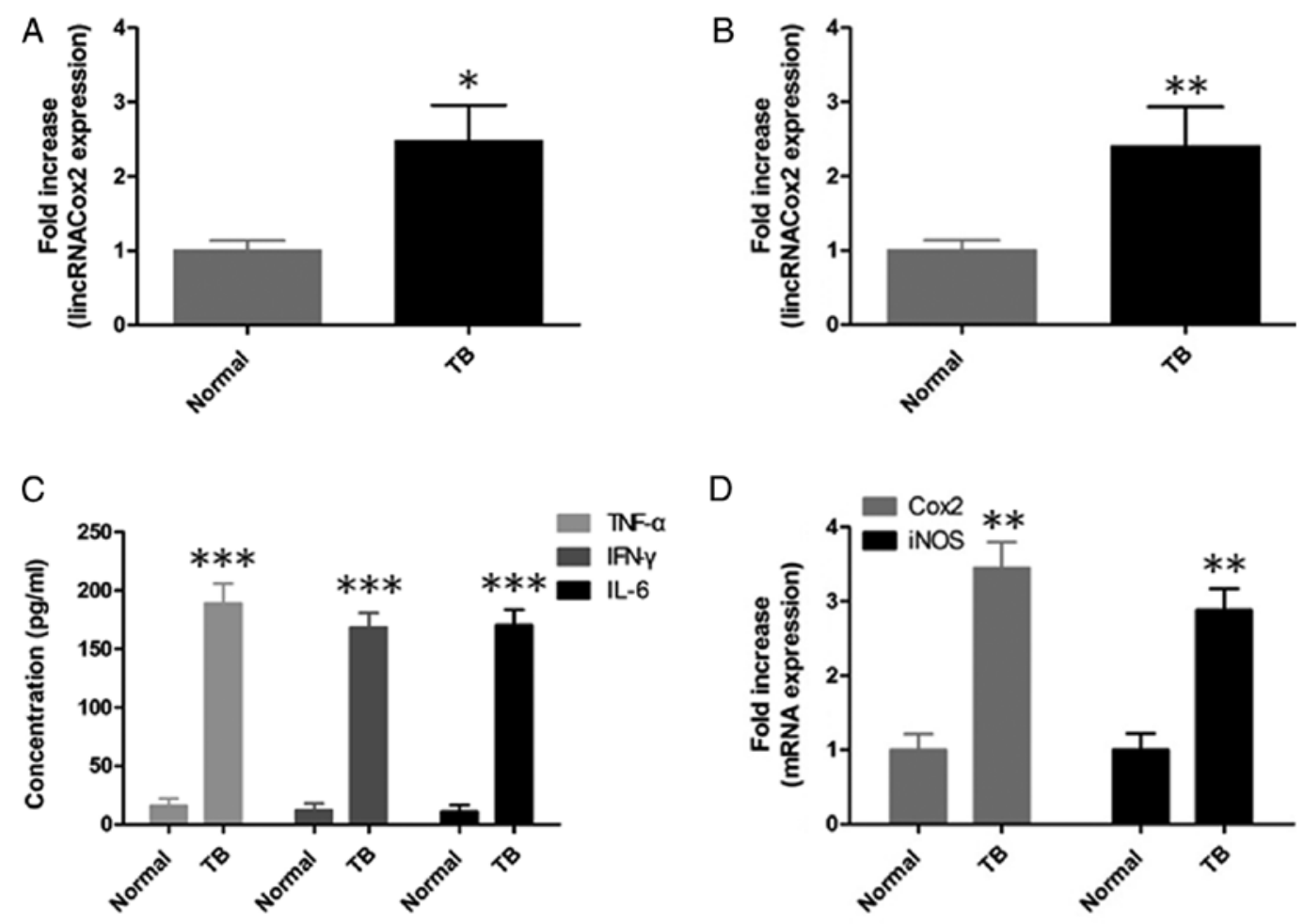

$\mathrm{D}$
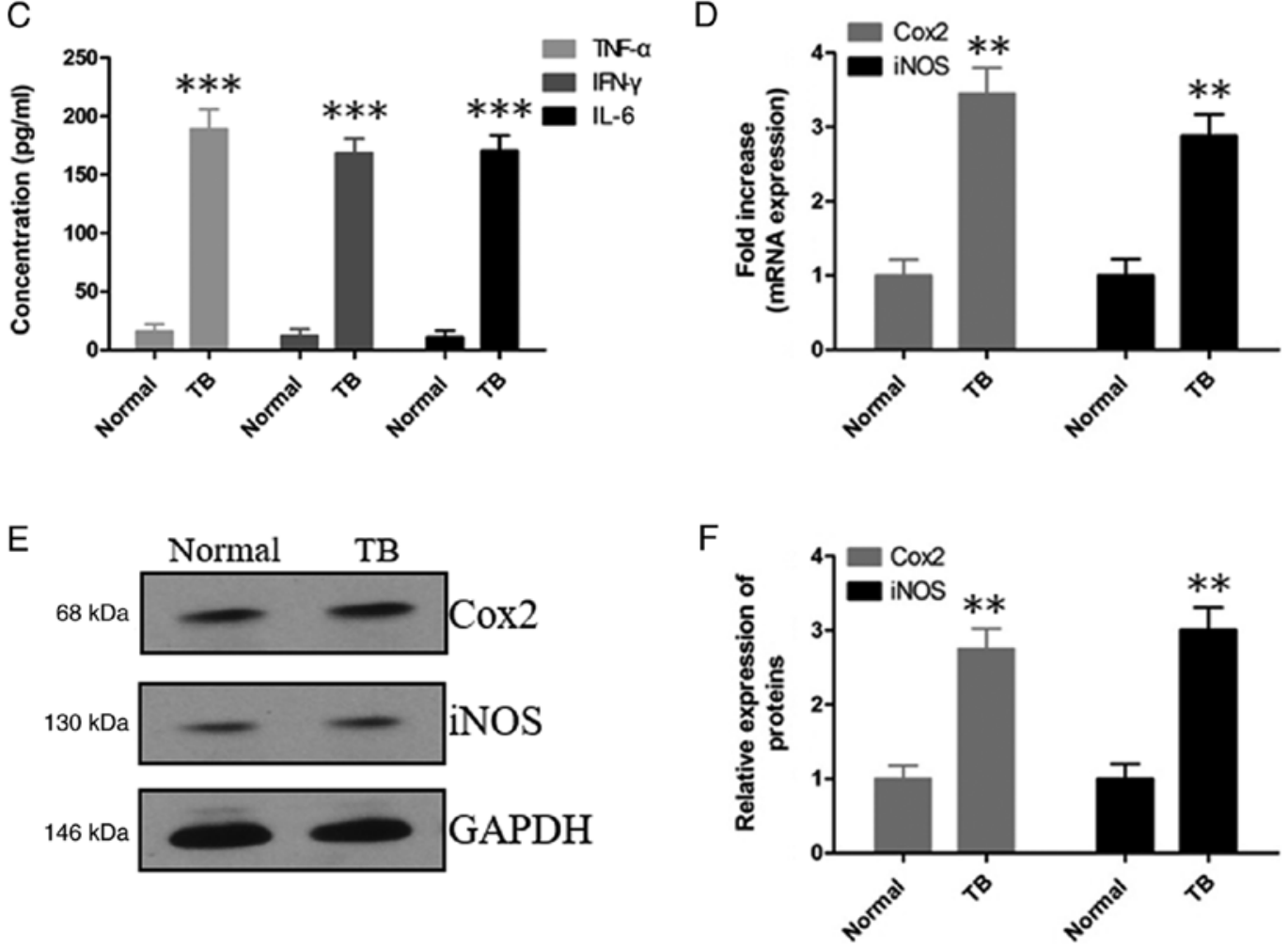

Figure 1. Expression level of lincRNACox 2 and the inflammatory response in the peripheral blood of patients with TB. RT-qPCR results of the expression level of lincRNACox 2 in the (A) plasma and (B) mononuclear cells of patients with TB. (C) ELISA was used to detect TNF- $\alpha$, IFN- $\gamma$ and IL-6 in the plasma of patients with TB. (D) RT-qPCR results of the mRNA expression levels of Cox2 and iNOS in mononuclear cells of patients with TB. (E) Protein expression levels and (F) analysis of Cox 2 and iNOS in mononuclear cells of patients with TB were measured by western blotting. Data are presented as the mean \pm SEM from three independent experiments, ${ }^{*} \mathrm{P}<0.05,{ }^{* *} \mathrm{P}<0.01$ and ${ }^{* * *} \mathrm{P}<0.001$ vs. Normal. RT-qPCR, reverse transcription-quantitative PCR; lincRNACox 2, long intergenic non-coding cyclooxygenase 2 RNA; TNF, tumor necrosis factor; IFN, interferon; IL, interleukin; TB, tuberculosis; iNOS, inducible NO synthase.

were infected with H37Ra $\left(1 \times 10^{5} \mathrm{CFU}\right)$ for $24 \mathrm{~h}$. For titration of intracellular H37Ra, macrophages were lysed with $0.025 \%$ SDS, neutralized with $0.5 \%$ albumin and grow on Middlebrooks 7H9 plates (cat. no. GOY-PYJ0567; Shanghai Guyan Biotechnology Company). Then, H37Ra titration was determined after 3, 6, 9, 12 and 15 days to analyze their proliferative capacity by bacteria plate count.

Statistical analysis. Data are presented as the mean \pm SEM from $\geq 3$ independent experiments. Data were compared with an unpaired Student's t-test or one-way analysis of variance with Tukey's post hoc test. All statistical tests were performed with GraphPad Prism 6.0 (GraphPad Software, Inc.). P $<0.05$ was considered to indicate a statistically significant difference.

\section{Results}

Expression of lincRNACox 2 and inflammatory responses were analyzed in patients with $T B$. lincRNACox 2 is a critical gene for regulating the expression level of NF- $\kappa \mathrm{B}$ in macrophages and NF- $\mathrm{KB}$ is an important protein regulating the inflammatory pathway especially in the process of tuberculosis infection. Thus the expression of lincRNACox 2 was investigated in TB patients. The expression levels of lincRNACox2 were determined by RT-qPCR in the plasma (Fig. 1A) and mononuclear cells of patients with TB (Fig. 1B). It was found that lincRNACox 2 was significantly increased in plasma and mononuclear cells of patients with TB compared with healthy individuals. Moreover, the expression levels of TNF- $\alpha$, IFN- $\gamma$ and IL-6 in the plasma of patients with TB were significantly 

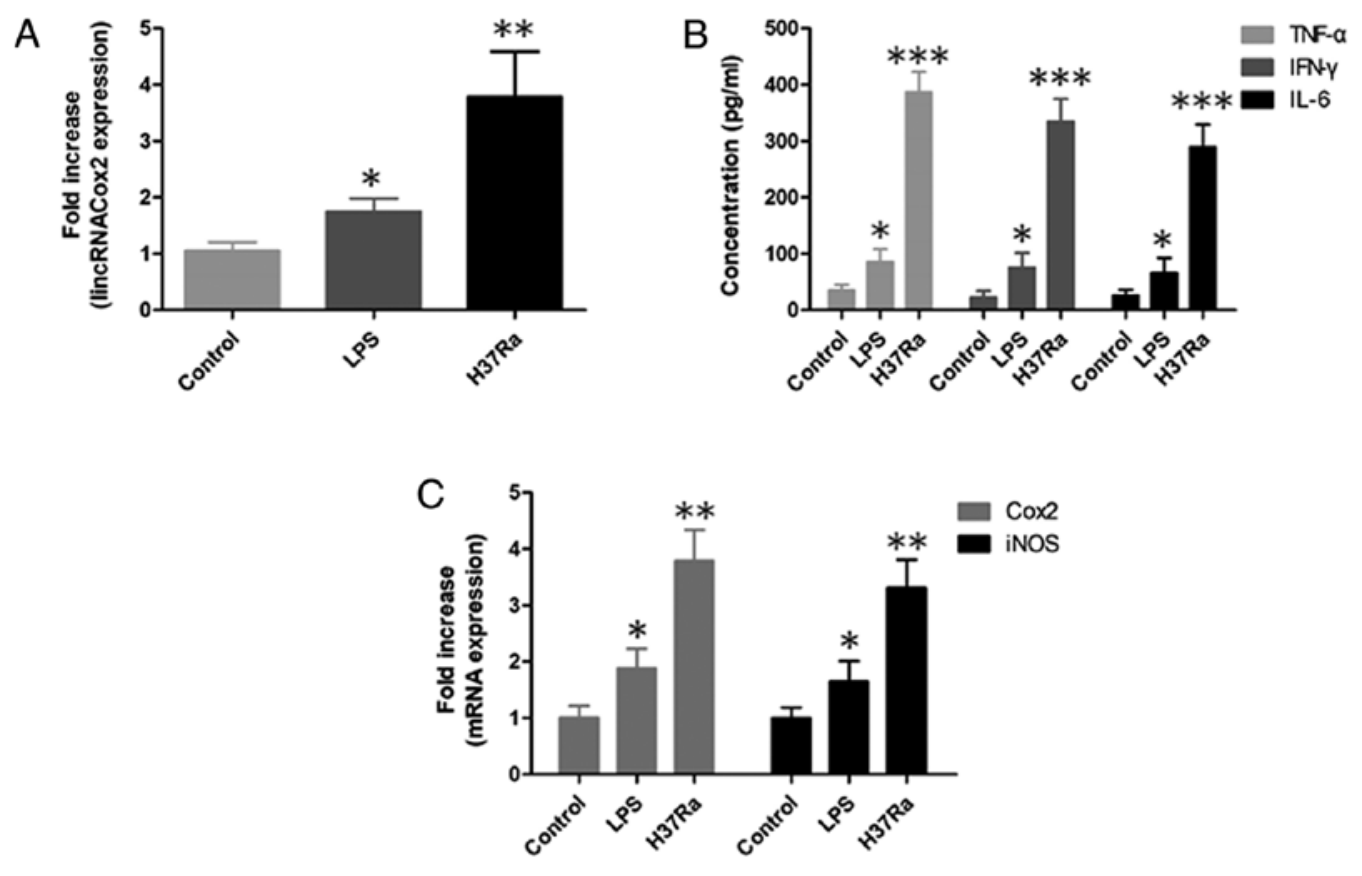

$\mathrm{D}$
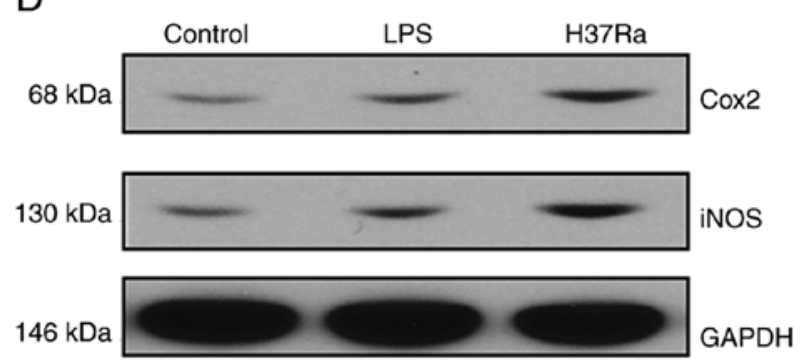

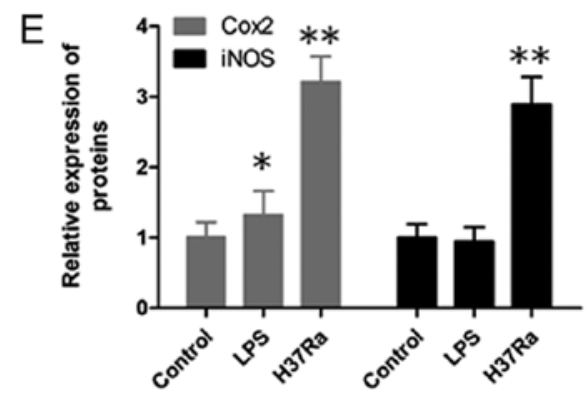

Figure 2. lincRNACox2 expression level and the inflammatory responses in H37Ra infected macrophages. (A) lincRNACox2 was detected in H37Ra infected macrophages by RT-qPCR. (B) Expression levels of TNF- $\alpha$, IFN- $\gamma$ and IL- 6 in the cell supernatant of H37Ra infected macrophages were determined by ELISA. (C) mRNA expression levels of Cox 2 and iNOS in H37Ra infected macrophages were measured by RT-qPCR. (D) Protein expression levels and (E) analysis of Cox 2 and iNOS in H37Ra infected macrophages were determined by western blotting. Data are presented as the mean \pm SEM from three independent experiments, ${ }^{*} \mathrm{P}<0.05,{ }^{* *} \mathrm{P}<0.01$ and ${ }^{* * * *} \mathrm{P}<0.001$ vs. Control. RT-qPCR, reverse transcription-quantitative PCR; lincRNACox 2 , long intergenic non-coding cyclooxygenase 2 RNA; TNF, tumor necrosis factor; IFN, interferon; IL, interleukin; iNOS, inducible NO synthase.

increased compared with healthy individuals (Fig. 1C). In addition, the expression levels of Cox 2 and iNOS in mononuclear cells of patients with TB were significantly elevated both at mRNA (Fig. 1D) and protein levels (Fig. 1E and F).

Expression of lincRNACox2 and inflammatory responses in H37Ra infected macrophages. Previous studies have shown that lincRNACox 2 increased significantly in TB patients and Cox 2 and iNOS were also promoted $(15,16)$, so the function of lincRNACox 2 in an in vitro cell experiment was assessed. In vitro cell experiments, RT-qPCR and western blotting were performed to assess the expression level of lincRNACox 2 in macrophages after exposure to H37Ra. It was demonstrated that lincRNACox 2 was increased in H37Ra infected macrophages (Fig. 2A). Furthermore, the inflammatory factors TNF- $\alpha$, IFN- $\gamma$ and IL-6 were increased in the supernatant of H37Ra infected macrophages, as determined by ELISA (Fig. 2B). Moreover, the mRNA and protein expression levels of Cox 2 and iNOS were significantly increased in H37Ra infected macrophages compared with the control group (Fig. 2C-E).
lincRNACox 2 knockdown in macrophages. The expression of lincRNACox 2 was enhanced in TB patients and TB infection macrophages in vivo and in vitro, then the effects of the knockdown of lincRNACox 2 in TB infected host cells will be studied. The siRNA in the present study was designed to target lincRNACox 2 and lincRNACox 2 was transfected into macrophages to induce its overexpression. lincRNACox 2 was knocked down using an RNA interference approach and the expression level of lincRNACox 2 was measured by RT-qPCR. RACE PCR analysis results identified a transcript of lincRNACox 2 in macrophages using a luciferase reporter vector (Fig. 3A). Furthermore, it was found that the expression level of lincRNACox 2 was significantly decreased when macrophages were transfected with si-lincRNACox 2 (Fig. 3B). However, H37Ra infection promoted the expression level of lincRNACox 2 in si-lincRNACox 2 transfected macrophages (Fig. 3C).

Inflammatory responses in lincRNACox 2 knockdown macrophages are induced by H37Ra. As lincRNACox 2 plays a 


\section{A LincRNACox2}
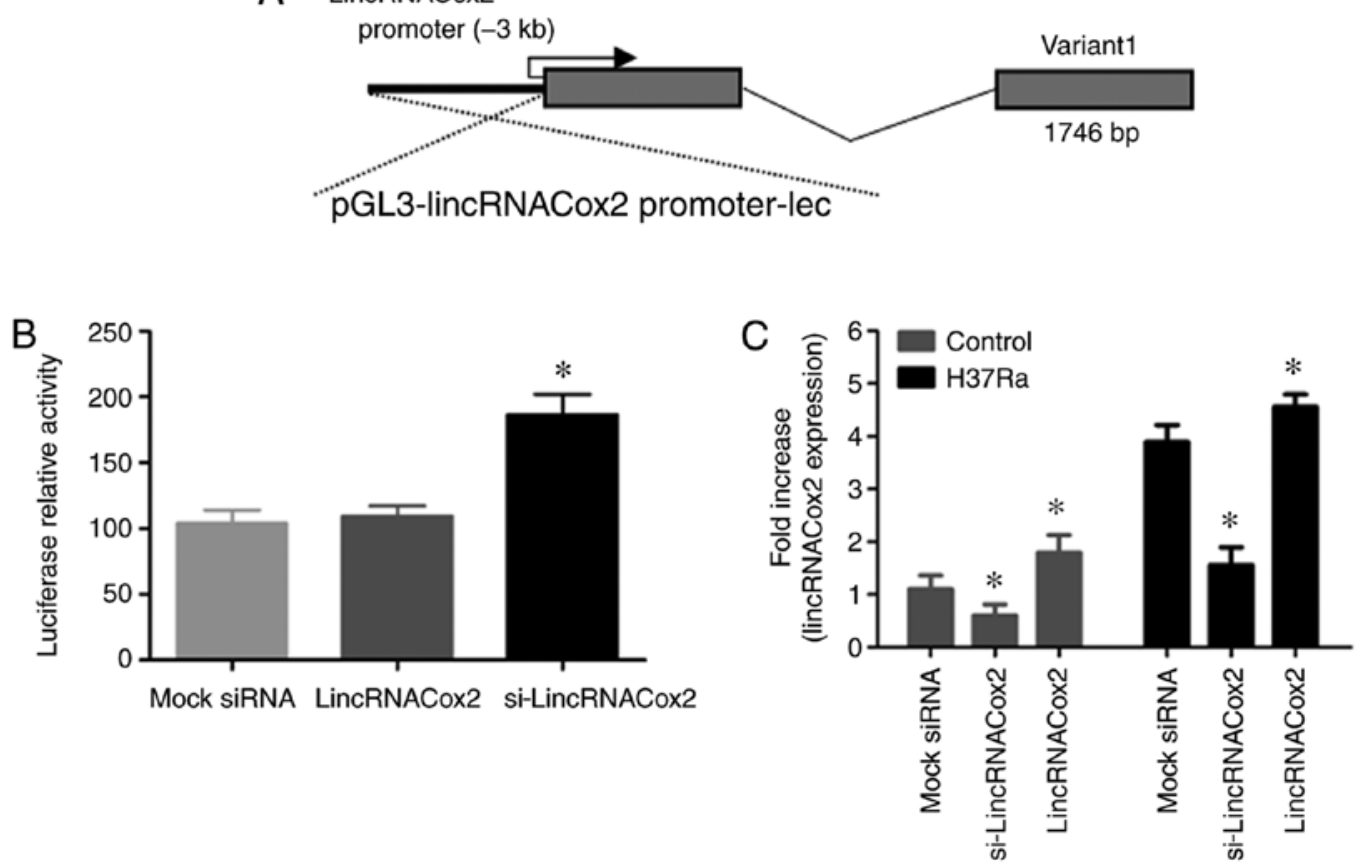

Figure 3. Luciferase reporter assay of lincRNACox 2 promoter infected macrophages. (A) Upstream sequence of lincRNACox 2 ( $3 \mathrm{~kb})$ was cloned and inserted into the pGL3-luciferase construct. (B) Luciferase activity assay results indicated that macrophages infected with si-lincRNACox 2 significantly increased luciferase activity. (C) Expression level of lincRNACox2 in H37Ra infected macrophages transfected with siRNA were determined by RT-qPCR. Data are presented as the mean \pm standard error of the mean from three independent experiments, ${ }^{*} \mathrm{P}<0.05$ vs. Mock siRNA. RT-qPCR, reverse transcription-quantitative PCR; siRNA, small interfering RNA; lincRNACox2, long intergenic non-coding cyclooxygenase 2 RNA.

A

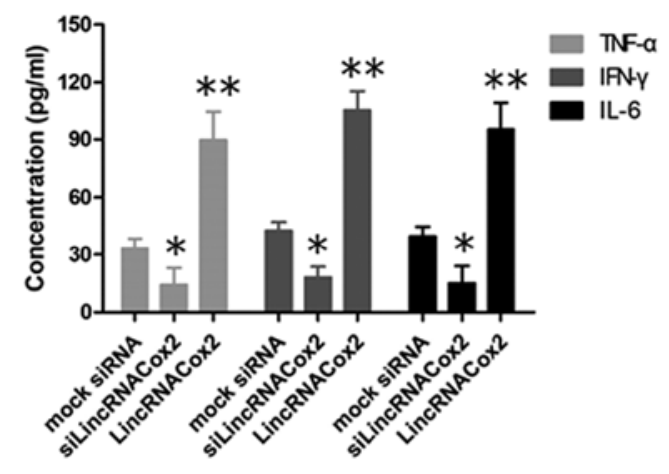

C

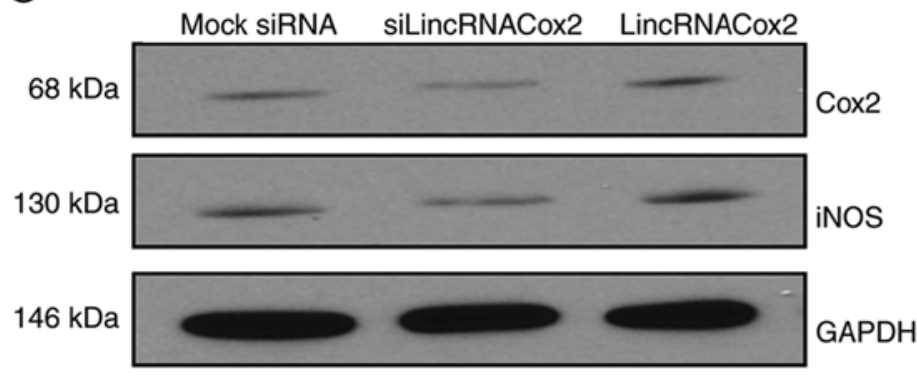

B
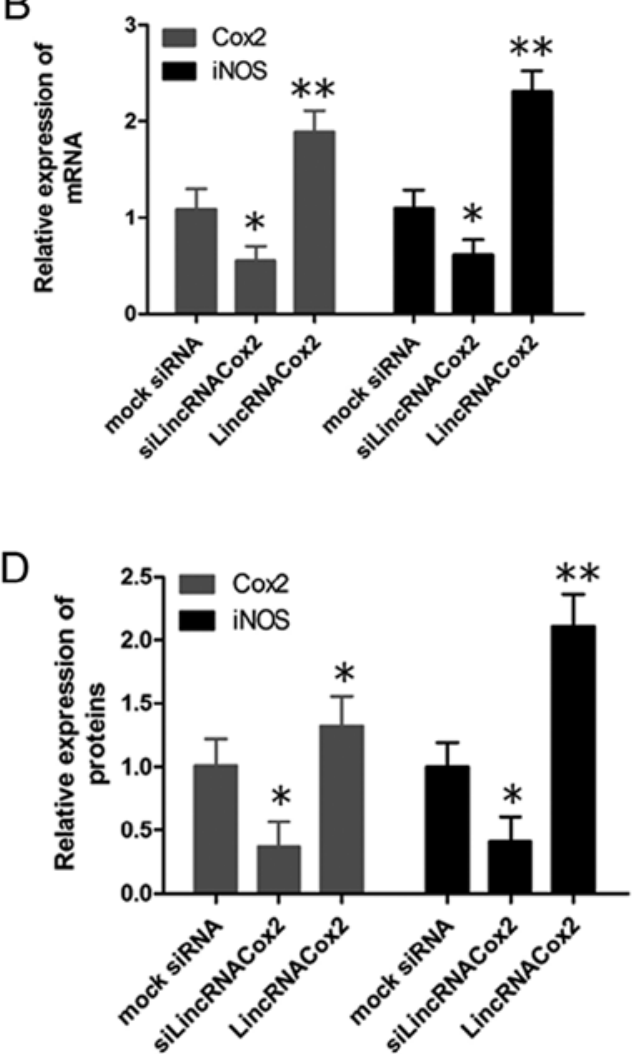

Figure 4. Inflammatory responses of H37Ra infected macrophages with lincRNACox 2 knockdown. (A) Levels of TNF- $\alpha$, IFN- $\gamma$ and IL- 6 in the supernatant of H37Ra infected macrophages with lincRNACox 2 knockdown were determined by ELISA. (B) mRNA expression levels of Cox 2 and iNOS in H37Ra infected macrophages with lincRNACox 2 knockdown were detected by reverse transcription-quantitative PCR. (C) Protein expression levels and (D) analysis of Cox2 and iNOS in H37Ra infected macrophages with lincRNACox 2 knockdown were determined by western blotting. Data are presented as the mean \pm standard error of the mean from three independent experiments. " $\mathrm{P}<0.05$ and ${ }^{* *} \mathrm{P}<0.01$ vs. Mock siRNA. lincRNACox 2 , long intergenic non-coding cyclooxygenase 2 RNA; TNF, tumor necrosis factor; IFN, interferon; IL, interleukin; iNOS, inducible NO synthase. 
A

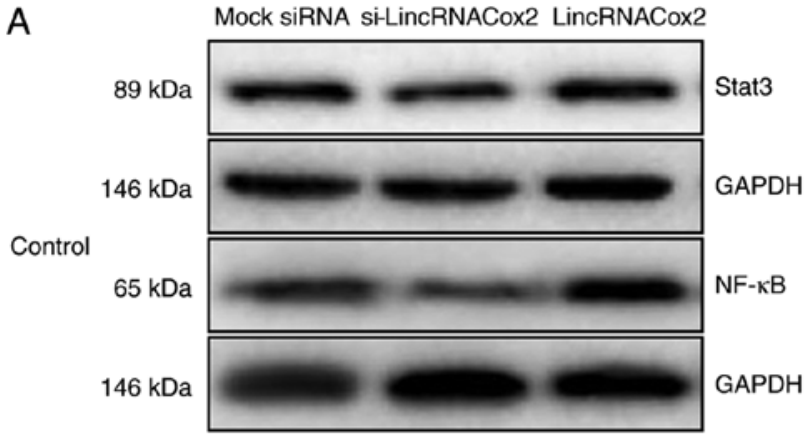

C

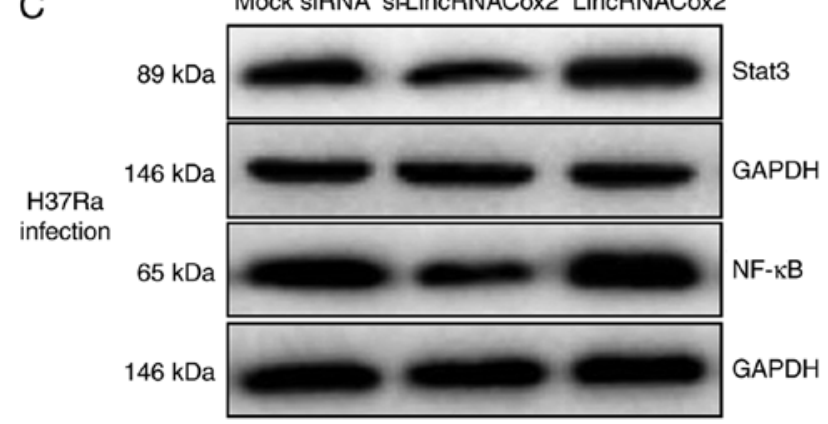

B
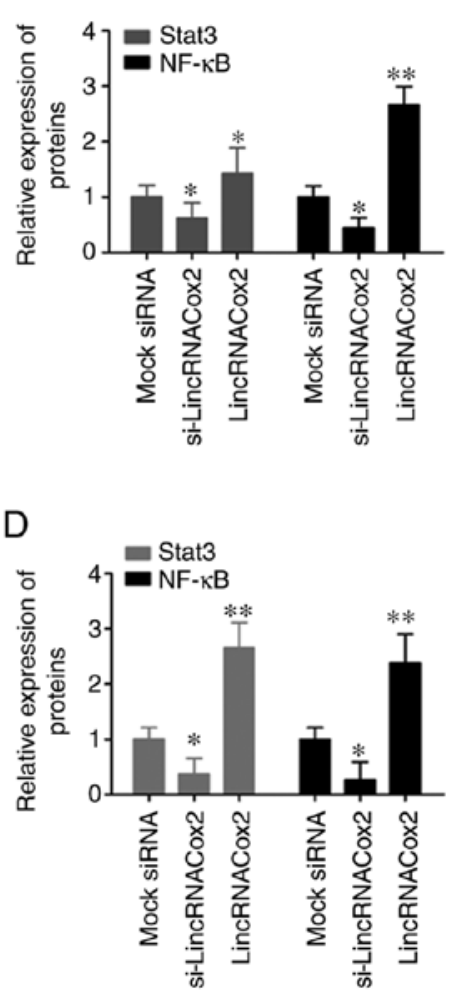

Figure 5. Inflammatory regulatory proteins Stat3 and NF- $\kappa$ B in H37Ra infected macrophages transfected with si-lincRNACox2. (A) Protein expression levels and (B) analysis of Stat 3 and NF- $\mathrm{kB}$ in lincRNACox 2 knockdown macrophages were determined by western blotting. (C) Protein expression levels and (D) analysis of Stat3 and NF-кB in lincRNACox 2 knockdown H37Ra infected macrophages were determined by western blotting. Data are presented as the mean \pm SEM from three independent experiments. "P<0.05 and ${ }^{* *} \mathrm{P}<0.01$ vs. Mock siRNA. lincRNACox2, long intergenic non-coding cyclooxygenase 2 RNA; siRNA, small interfering RNA.

very important role in the inflammatory response pathway, so the inflammatory response of TB infected macrophages in which lincRNACox 2 was knocked down was studied. After lincRNACox 2 was silenced, macrophages were infected with H37Ra. It was demonstrated that macrophages transfected with si-lincRNACox 2 had significantly decreased expression levels of the inflammatory factors TNF- $\alpha$, IFN- $\gamma$ and IL-6 compared with other groups (Fig. 4A). Moreover, knockdown of lincRNACox 2 reduced the mRNA and protein expression levels of Cox 2 and iNOS in H37Ra infection macrophages (Fig. 4B-D). Therefore, the present results suggested that the knockdown of lincRNACox 2 suppressed the inflammatory response in $\mathrm{H} 37 \mathrm{Ra}$ infected macrophages.

Inflammatory regulatory proteins Stat 3 and $N F-\kappa B$ are regulated by lincRNACox2. lincRNACox 2 regulates the expression of inflammatory cytokines and inflammatory regulating proteins, so the inflammatory signaling pathways such as Stat 3 and NF- $\kappa$ B associated with lincRNACox 2 were investigated. It was found that the knockdown of lincRNACox 2 significantly inhibited the protein expression levels of NF- $\kappa \mathrm{B}$ and Stat3 in macrophages (Fig. 5A and B). Moreover, H37Ra significantly suppressed the expression levels of the inflammatory regulatory proteins Stat 3 and NF- $\kappa$ B in lincRNACox 2 knockdown macrophages (Fig. 5C and D). Thus, the present results indicated that the $\mathrm{NF}-\kappa \mathrm{B}$ pathway may be triggered by lincRNACox 2 in macrophages. However, H37Ra is dependent on the expression level of lincRNACox 2 to activate the NF- $\kappa \mathrm{B}$ signaling pathway to induce a cellular inflammatory response.

Effect of lincRNACox2 on the apoptosis of H37Ra infected macrophages and the proliferation of H37Ra. lincRNACox2 could regulate the inflammatory response of TB infection macrophages through Stat 3 and $\mathrm{NF}-\kappa \mathrm{B}$ signaling pathways, however the influences of lincRNACox 2 on the apoptosis of H37Ra infected macrophages and the proliferation of H37Ra remains unknown. Macrophages transfected with si-lincRNACox 2 or lincRNACox 2 were infected with $\mathrm{H} 37 \mathrm{Ra}$ and the effect of lincRNACox 2 on the apoptosis and proliferation of H37Ra infected macrophages was investigated. It was demonstrated that knockdown of lincRNACox 2 significantly increased the apoptotic rate of H37Ra infect macrophages. However, the apoptotic rate of H37Ra infected macrophages with lincRNACox 2 overexpression was significantly decreased compared with control groups (Fig. 6A). Moreover, knockdown of lincRNACox 2 promoted the proliferation of H37Ra extracted from macrophages, but the overexpression of lincRNACox 2 inhibited the proliferation of H37Ra, suggesting an additive regulating effect of lincRNACox 2 on H37Ra infection (Fig. 6B). Collectively, the present results suggested that the overexpression of lincRNACox 2 in H37Ra infected macrophages could suppress H37Ra proliferation via various mechanisms, including cytokine production and oxygen/nitrogen metabolism (Fig. 7). 

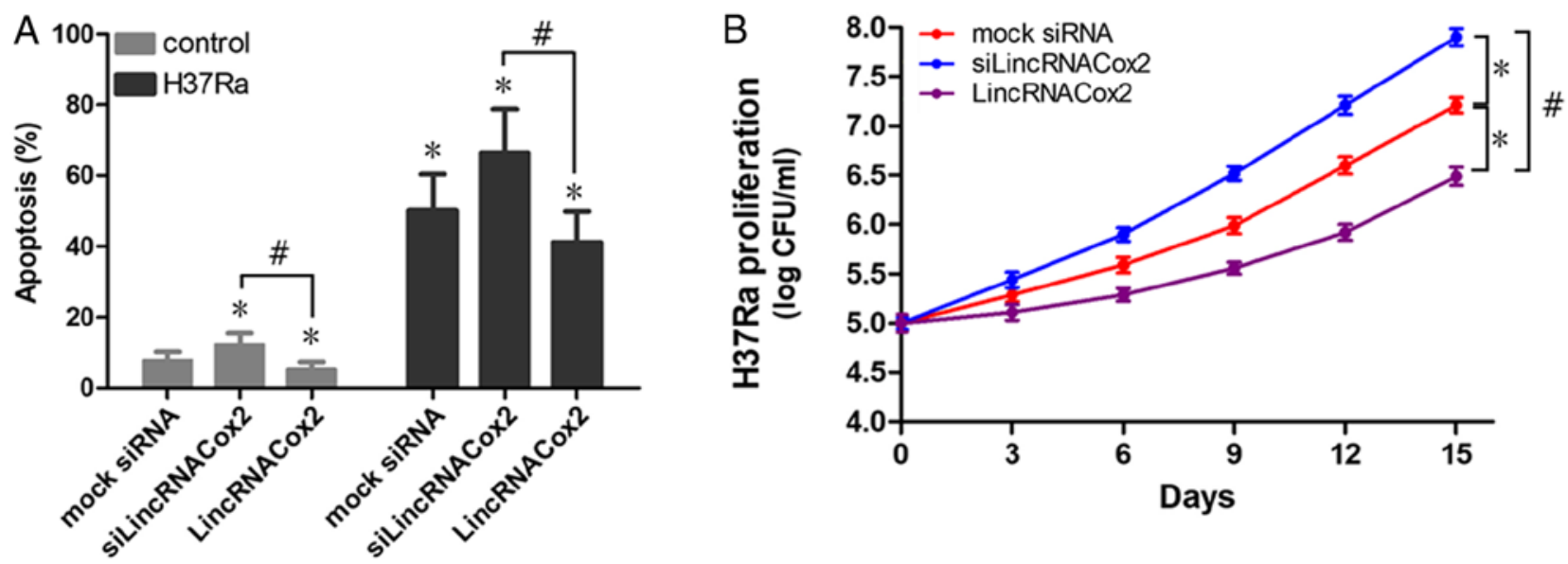

Figure 6. Effect of lincRNACox 2 knockdown on the apoptosis of H37Ra infected macrophages and the proliferation of H37Ra in macrophages. (A) Apoptotic rate of H37Ra infected macrophages transfected with si-lincRNACox 2 was analyzed by flow cytometry. (B) Proliferation of H37Ra from infection macrophages transfected with si-lincRNACox 2 was assessed by bacteria plate count. Data are presented as the mean \pm SEM from three independent experiments, ${ }^{*} \mathrm{P}<0.05$ vs. mock siRNA; ${ }^{\#} \mathrm{P}<0.05$. lincRNACox 2 , long intergenic non-coding cyclooxygenase 2 RNA; siRNA, small interfering RNA; CFU, colony forming units.

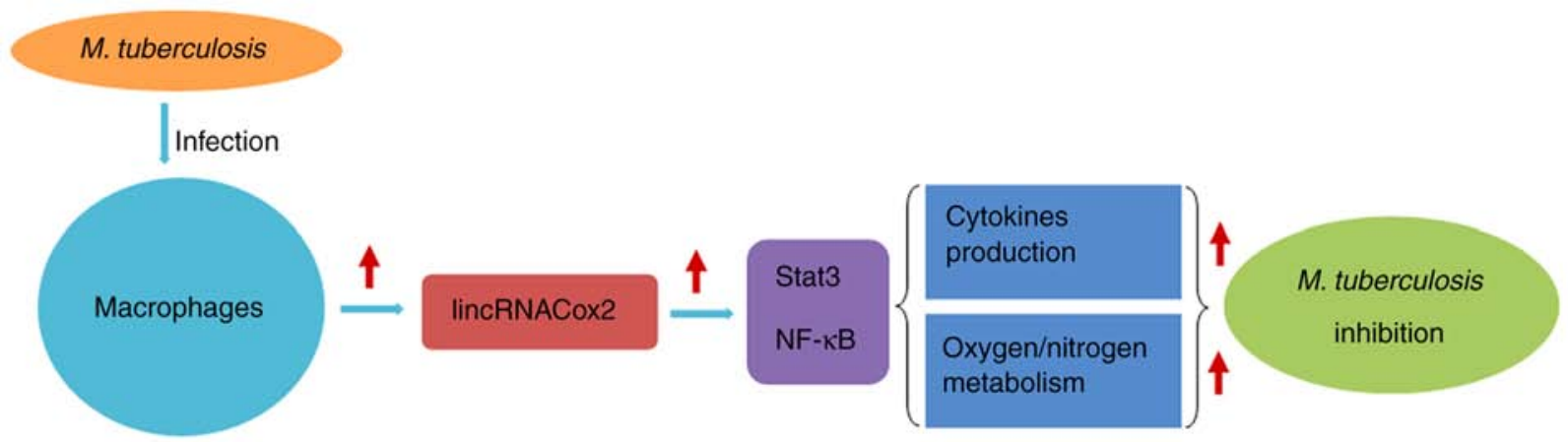

Figure 7. Schematic illustration of lincRNACox2-mediated macrophage inhibition of H37Ra proliferation. Overexpression of lincRNACox 2 after H37Ra infection suppressed the proliferation of H37Ra via various mechanisms, including cytokines production and enhancement of oxygen/nitrogen metabolism. lincRNACox2, long intergenic non-coding cyclooxygenase 2 RNA.

\section{Discussion}

Previous studies have showed that lncRNAs play significant roles in various biological processes, such as the immune response, tumorigenesis and cellular infectious diseases (17-20). Interactions between macrophages and M. tuberculosis can alter genetic material expression profiles, including circular RNAs expression profiles and lncRNA expression profiles. Examining these changes can improve the understanding of the regulation of anti-mycobacterium immunity in macrophages $(21,22)$. Furthermore, previous studies have demonstrated IncRNAs can be used as biomarkers or therapeutic targets for various diseases $(23,24)$. Several IncRNAs, such as miR3945HG-V1 and miR3945HG-V2, are differentially expressed in H37Ra or H37Rv infected macrophages, and could be used as novel diagnostic biomarkers for TB. Thus, this would provide insight into the mechanisms of $M$. tuberculosis macrophage interactions and identify potential targets for the diagnosis and treatment of TB (25). LncRNA-TNF and HNRNPL related immunoregulatory lncRNA in THP-1 macrophages can modulate the expression levels of TNF- $\alpha$ and other inflammatory genes to regulate inflammation (26). Moreover, lincRNA-exopolysaccharide can regulate the inflammatory response in macrophages exposed to microbial ligands via the regulation of interferon regulated gene expression (27). Furthermore, lincRNACox2 can mediate both the activation and repression of different immune genes.

The present results suggested that lincRNACox 2 was increased in patients with TB and H37Ra infected macrophages, and that the inflammatory factors TNF- $\alpha$, IFN- $\gamma$, IL- 6 , Cox 2 and iNOS were increased by lincRNACox 2 in vivo and in vitro. Thus, lincRNACox 2 may be a critical mediator for the development of inflammatory responses in both microglia and macrophages. The present study knocked down the expression of lincRNACox 2 in H37Ra infected macrophages and found this could suppress the inflammatory factors TNF- $\alpha$, IFN- $\gamma$, IL-6, Cox 2 and iNOS in these macrophages, which indicated that lincRNACox 2 may regulate the inflammatory response of H37Ra infected macrophages.

lincRNACox 2 expression is influenced by NF- $\mathrm{kB}$ signaling and serves as a coactivator of transcriptional factors to regulate inflammatory responses (28). NF- $\mathrm{kB}$ is composed of homo- or heterodimeric complexes of NF- $\mathrm{KB}$ subunits including $\mathrm{p} 65$, RelB, c-Rel, p50 and p52 in humans, and is an important regulator of inflammatory and immune responses (29). However, 
the dysregulation of the NF- $\mathrm{NB}$ signaling pathway is linked to cancer, inflammation, autoimmune diseases and infectious diseases $(30,31)$. Furthermore, numerous acute and late proinflammatory genes, critical to the pathogenesis of sepsis, are controlled by the $\mathrm{NF}-\kappa \mathrm{B}$-gene (32). A nuclear $\mathrm{NF}-\kappa \mathrm{B}$ paradox has been identified during severe systemic inflammation, as defined by a disassociation between cytosolic $\mathrm{NF}-\kappa \mathrm{B}$ activation and nuclear NF- $\kappa \mathrm{B}$ transcriptional processes (33). Previous studies have demonstrated the inflammatory response is induced in macrophages due to lipopolysaccharide stimulation via the $\mathrm{NF}-\kappa \mathrm{B}$ signaling pathway $(34,35)$.

In the present study, the inflammatory response related signaling pathway involving Stat 3 and $N F-\kappa B$ was analyzed in H37Ra infected macrophages transfected with si-lincRNACox2. The present results suggested that the expression of lincRNACox 2 is induced by the NF- $\mathrm{NB}$ signaling pathway, potentially via binding of $\mathrm{NF}-\kappa \mathrm{B}$ subunits to the promoter region of the gene locus. The induction of lincRNACox 2 in patients with TB and H37Ra infected macrophages, along with the expression of the inflammatory factors TNF- $\alpha$, IFN- $\gamma$, IL-6, Cox 2 and iNOS, suggested that NF- $\kappa$ B-mediated lincRNACox 2 expression and its subsequent impact on the transcription of inflammatory genes may occur in vivo during systemic inflammation. In addition, knockdown of lincRNACox 2 promoted the proliferation of H37Ra via the inflammatory pathway or oxygen/nitrogen metabolism, thus indicating that lincRNACox 2 may have a central role in M. tuberculosis infection.

In conclusion, the present results suggested that lincRNACox 2 may be used as a novel biomarker for the diagnosis and therapeutic target of TB. However, the function of lincRNACox 2 in regulating the immune response of macrophages infected with H37Ra is still not fully understood.

\section{Acknowledgements}

Not applicable.

\section{Funding}

No funding was received.

\section{Availability of data and materials}

The datasets used and/or analyzed during the current study are available from the corresponding author on reasonable request.

\section{Authors' contributions}

DL and YZ designed the experiments; $C G$ and $L Z$ performed the experiments and analyzed the data; DL and YZ drafted and revised the manuscript. All authors read and approved the final manuscript.

\section{Ethics approval and consent to participate}

The present study was approved by the Ethics Committee of the China-Japan Friendship Hospital (grant no. ZRYYEC/2015/27-2). Written informed consent was obtained from all donors prior to the study.

\section{Patient consent for publication}

Not applicable.

\section{Competing interests}

The authors declare that they have no competing interests.

\section{References}

1. Zheng L, Leung E, Lee N, Lui G, To KF, Chan RC and Ip M: Differential microRNA expression in human macrophages with Mycobacterium tuberculosis infection of Beijing/W and non-Beijing/W strain types. PLoS One 10: e0126018, 2015.

2. Suárez I, Fünger SM, Kröger S, Rademacher J, Fätkenheuer G and Rybniker J: The diagnosis and treatment of tuberculosis. Dtsch Arztebl Int 116: 729-735, 2019.

3. Baer CE, Rubin EJ and Sassetti CM: New insights into TB physiology suggest untapped therapeutic opportunities. Immunol Rev 264: 327-343, 2015.

4. HmamaZ,Peña-DíazS,Joseph S and Av-Gay Y:Immunoevasion and immunosuppression of the macrophage by Mycobacterium tuberculosis. Immunol Rev 264: 220-232, 2015.

5. Huang Z, Luo Q, Guo Y, Chen J, Xiong G, Peng Y, Ye J and Li J: Mycobacterium tuberculosis-induced polarization of human macrophage orchestrates the formation and development of tuberculous granulomas in vitro. PLoS One 10: e0129744, 2015.

6. Korb VC, Chuturgoon AA and Moodley D: Mycobacterium tuberculosis: Manipulator of protective immunity. Int J Mol Sci 17: 131,2016

7. Heward JA and Lindsay MA: Long non-coding RNAs in the regulation of the immune response. Trends Immunol 35: 408-419, 2014.

8. Bartonicek N, Maag JL and Dinger ME: Long noncoding RNAs in cancer: Mechanisms of action and technological advancements. Mol Cancer 15: 43, 2016.

9. Zhao Z, Zhang M, Ying J, Hu X, Zhang J, Zhou Y, Zhou Y, Song $X$ and Ying B: Significance of genetic polymorphisms in long non-coding RNA AC079767.4 in tuberculosis susceptibility and clinical phenotype in western Chinese Han population. Sci Rep 7: 965, 2017.

10. Mirza AH, Kaur S, Brorsson CA and Pociot F: Effects of GWAS-associated genetic variants on lncRNAs within IBD and T1D candidate loci. PLoS One 9: e105723, 2014.

11. He J, Ou Q, Liu C, Shi L, Zhao C, Xu Y, Kong SK, Loo JFC, Li B and Gu D: Differential expression of long non-coding RNAs in patients with tuberculosis infection. Tuberculosis (Edinb) 107: 73-79, 2017.

12. Fu Y, Gao K, Tao E, Li R and Yi Z: Aberrantly expressed long non-coding RNAs in $\mathrm{CD}^{+} \mathrm{T}$ cells response to active tuberculosis. J Cell Biochem 118: 4275-4284, 2017.

13. Pawar K, Hanisch C, Palma Vera SE, Einspanier R and Sharbati S: Down regulated lncRNA MEG3 eliminates mycobacteria in macrophages via autophagy. Sci Rep 6: 19416, 2016.

14. Livak KJ and Schmittgen TD: Analysis of relative gene expression data using real-time quantitative PCR and the 2(-Delta Delta C(T)) method. Methods 25: 402-408, 2001.

15. Carpenter S, Aiello D, Atianand MK, Ricci EP, Gandhi P, Hall LL, Byron M, Monks B, Henry-Bezy M, Lawrence JB, et al: A long noncoding RNA mediates both activation and repression of immune response genes. Science 341: 789-792, 2013.

16. Lin J, Jiang Y, Liu D, Dai X, Wang M and Dai Y: Early secreted antigenic target of 6-kDa of Mycobacterium tuberculosis induces transition of macrophages into epithelioid macrophages by downregulating Inos/NO-mediated H3K27 trimethylation in macrophages. Mol Immunol 117: 189-200, 2020.

17. Wang KC and Chang HY: Molecular mechanisms of long noncoding RNAs. Mol Cell 43: 904-914, 2011.

18. Prensner JR and Chinnaiyan AM: The emergence of lncRNAs in cancer biology. Cancer Discov 1: 391-407, 2011.

19. Moran VA, Perera RJ and Khalil AM: Emerging functional and mechanistic paradigms of mammalian long non-coding RNAs. Nucleic Acids Res 40: 6391-6400, 2012.

20. Satpathy AT and Chang HY: Long noncoding RNA in hematopoiesis and immunity. Immunity 42: 792-804, 2015. 
21. Rajaram MV, Ni B, Dodd CE and Schlesinger LS: Macrophage immunoregulatory pathways in tuberculosis. Semin Immunol 26 : 471-485, 2014

22. Gan H, Lee J, Ren F, Chen M, Kornfeld H and Remold HG: Mycobacterium tuberculosis blocks crosslinking of annexin-1 and apoptotic envelope formation on infected macrophages to maintain virulence. Nat Immunol 9: 1189-1197, 2008.

23. Boon RA, Jae N, Holdt L and Dimmeler S: Long noncoding RNAs: From clinical genetics to therapeutic targets? J Am Coll Cardiol 67: 1214-1226, 2016.

24. Shao Y, Ye M, Li Q, Sun W, Ye G, Zhang X, Yang Y, Xiao B and Guo J: LncRNA-RMRP promotes carcinogenesis by acting as a miR-206 sponge and is used as a novel biomarker for gastric cancer. Oncotarget 7: 37812-37824, 2016.

25. Yang X, Yang J, Wang J, Wen Q, Wang H, He J, Hu S, He W, Du X, Liu S and Ma L: Microarray analysis of long noncoding RNA and mRNA expression profiles in human macrophages infected with Mycobacterium tuberculosis. Sci Rep 6: 38963, 2016.

26. Li Z, Chao TC, Chang KY, Lin N, Patil VS, Shimizu C, Head SR, Burns JC and Rana TM: The long noncoding RNA THRIL regulates TNF $\alpha$ expression through its interaction with hnRNPL. Proc Natl Acad Sci USA 111: 1002-1007, 2014.

27. Atianand MK, Hu W, Satpathy AT, Shen Y, Ricci EP, Alvarez-Dominguez JR, Bhatta A, Schattgen SA, McGowan JD, Blin J, et al: A long noncoding RNA lincRNA-EPS acts as a transcriptional brake to restrain inflammation. Cell 165: 1672-1685, 2016.

28. Hu G, Liao K, Niu F, Yang L, Dallon BW, Callen S, Tian C, Shu J, CuiJ,SunZ,etal: Astrocyte EV-induced lincRNA-Cox 2 regulates microglial phagocytosis: Implications for morphine-mediated neurodegeneration. Mol Ther Nucleic Acids 13: 450-463, 2018.

29. Hayden MS and Ghosh S: Shared principles in NF-kappaB signaling. Cell 132: 344-362, 2008.
30. Natoli G, Saccani S, Bosisio D and Marazzi I: Interactions of NF-kappaB with chromatin: The art of being at the right place at the right time. Nat Immunol 6: 439-445, 2005.

31. Haddad JJ and Abdel-Karim NE: NF- $\kappa$ B cellular and molecular regulatory mechanisms and pathways: Therapeutic pattern or pseudo regulation? Cell Immunol 271: 5-14, 2011.

32. Böhrer H, Qiu F, Zimmermann T, Zhang Y, Jllmer T, Männel D, Böttiger BW, Stern DM, Waldherr R, Saeger HD, et al: Role of NFkappaB in the mortality of sepsis. J Clin Invest 100: 972-985, 1997.

33. Schreiber J, Jenner RG, Murray HL, Gerber GK, Gifford DK and Young RA: Coordinated binding of NF-kappaB family members in the response of human cells to lipopolysaccharide. Proc Natl Acad Sci USA 103: 5899-5904, 2006.

34. Wang F, Zhang W, Wang C, Fang X, Cheng H, Liu S and Chen XL: Inhibitor of Tec kinase, LFM-A13, decreases pro-inflammatory mediators production in LPS-stimulated RAW264.7 macrophages via NF-кB pathway. Oncotarget 8: 34099-34110, 2017.

35. Chen Y, Guo S, Jiang K, Wang Y, Yang M and Guo M: Glycitin alleviates lipopolysaccharide-induced acute lung injury via inhibiting NF- $\mathrm{KB}$ and MAPKs pathway activation in mice. Int Immunopharmacol 75: 105749, 2019.

This work is licensed under a Creative Commons Attribution-NonCommercial-NoDerivatives 4.0 International (CC BY-NC-ND 4.0) License. 